\title{
CEEOG
}




\title{
Country: Central and Eastern Europe
}

\section{Group: Central and East European Oncology Group (CEEOG)}

\author{
Chair: J. Jassem \\ Medical University of Gdansk \\ Department of Oncology and Radiotherapy \\ Debinki St. 7 \\ 80-211 GDANSK \\ POLAND \\ Tel: +48583492270 \\ Fax: +48583492270 \\ Email: jjassem@amg.gda.pl \\ Biostatistics A. Badzio, R. Dziadziuszko \\ Unit: $\quad$ Medical University of Gdansk \\ Department of Oncology and Radiotherapy \\ Debinki St. 7 \\ 80-211 GDANSK \\ POLAND \\ Tel: +48583492256 \\ Fax: +48583492141 \\ Email: bodek@amg.gda.pl / rafald@post.pl
}

Study Center: A. Janczylo

Medical University of Gdansk

Department of Oncology and Radiotherapy

Debinki St. 7

80-211 GDANSK

POLAND

Tel: +48583492141

Fax: +48583492141

Email: a.janczylo@amg.gda.pl

Website: www.ceeog.org 